\title{
Spam Fried Rice
}

\section{Ian Roesler}

\author{
Spam \\ White Rice \\ Sesame oil or vegetable oil \\ Soy Sauce \\ Oyster sauce \\ Sambal oelek or chili garlic sauce (Green lid with a \\ rooster on the bottle is optional) \\ Snow peas
}

1. Put a skillet on medium-high heat. Whilst it warms up begin removing the Spam from its can and cut into bitesize cubes. When skillet is ready add enough sesame oil to cover bottom of pan then add Spam. Add soy and oyster sauces then cook until Spam is crispy. When done, remove Spam to a plate.

2. Cook snow peas in a similar manner with oil and sauces. However, any vegetable can be used. Cook until done. I cook the snow peas to where they still retain their crunch. Remove snow peas to a plate after done.

3. Take cooked white rice and add it in chunks. Turn up the heat to hot. Cook with oil and sauces. Constantly move rice around until done. It turns brown. If one desires, they may add their chili sauce now. When rice is done add back Spam and snow peas. Stir. Eat.

I like Spam. I like fried rice. So why not combine them? This recipe is a variation of a recipe that I got from a deck of playing cards with Spam recipes on them. I just eyeball the sauces. And I prepare twoand-a-half cups of dried basmati rice beforehand. 